\title{
Metastatic Prostate Carcinoma Presenting as a Superscan on 68Ga-PSMA PET/CT
}

Ismaheel Lawal, MD, Mariza Vorster, MD, PhD, Tebatso Boshomane, MD, Kehinde Ololade, MD, Thomas Ebenhan, PhD, and Mike Sathekge*, MD, PhD

University of Pretoria, Pretoria, South Africa.

Conflicts of interest and sources of funding: none declared.

*Correspondence to: Mike Sathekge, MD, PhD, Nuclear Medicine Department, University of Pretoria and Steve Biko Academic Hospital, Private Bag X169, Pretoria, 0001, South Africa. E-mail: mike.sathekge@up.ac.za

Abstract: We describe the finding of a metastatic superscan detected by ${ }^{68} \mathrm{Ga}-\mathrm{PSMA} P E T / C T$ imaging. A 63-year-old man with metastatic prostate carcinoma underwent ${ }^{68} \mathrm{Ga}-\mathrm{PSMA}$ $\mathrm{PET} / \mathrm{CT}$ imaging for staging and evaluation of the most appropriate therapeutic option. Images demonstrated diffuse and extensive skeletal uptake in the axial and appendicular skeleton, corresponding to the typical red marrow distribution. Intense soft tissue uptake was also seen in the prostate and multiple pelvic and abdominal lymph nodes.

Key Words: prostate carcinoma, ${ }^{68} \mathrm{Ga}-\mathrm{PSMA} \mathrm{PET} / \mathrm{CT}$, superscan
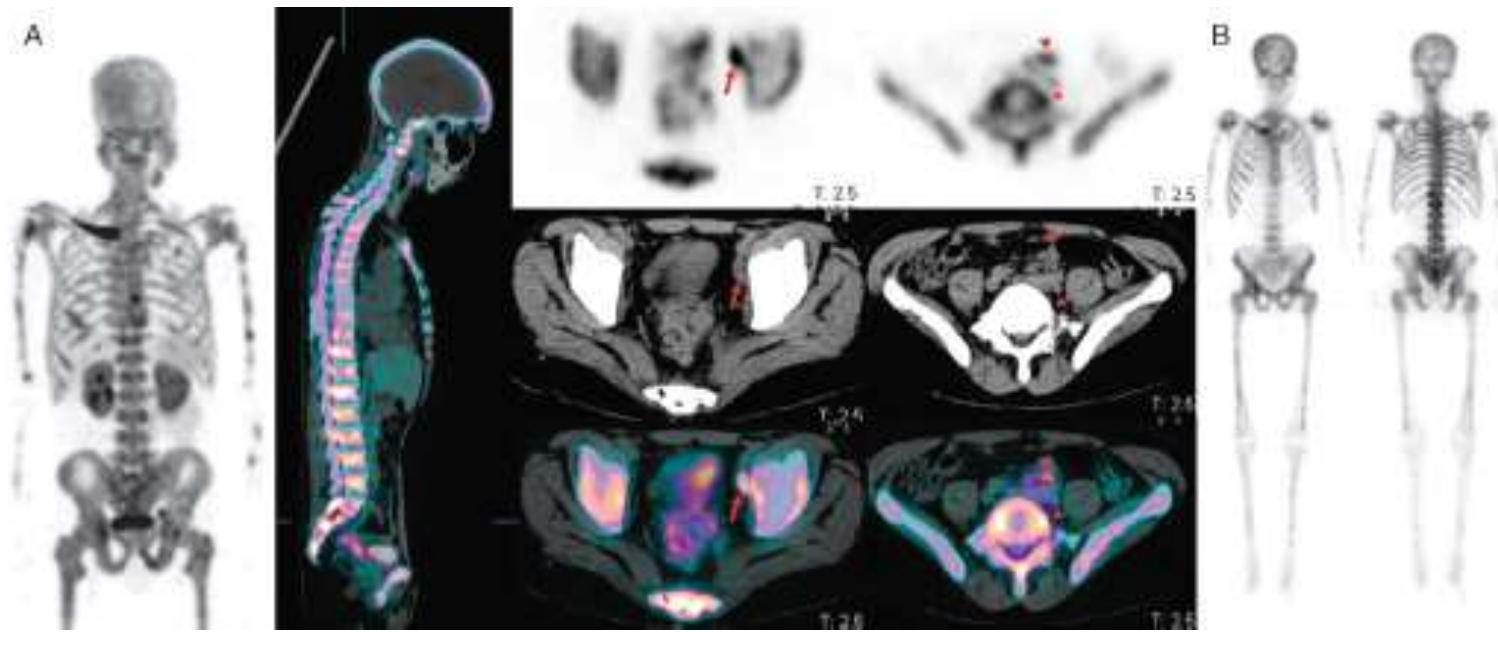

FIGURE 1. A 63-year-old male patient presented with metastatic prostate cancer. Histology demonstrated infiltrating adenocarcinoma and a Gleason score of $8(4+4)$ with perineural infiltration. The PSA was 100 $\mathrm{ng} / \mathrm{mL}$, which is predictive of the presence of multiple bone metastases. ${ }^{1}$ The patient was referred for ${ }^{68} \mathrm{Ga}$ PSMA PET/CT imaging (A), which revealed diffuse generalized skeletal tracer uptake as well as multiple traceravid abdominal (arrowhead) and pelvic (arrow) lymphadenopathy. The prostate also showed increased tracer accumulation. Prostate cancer commonly metastasizes to bone ${ }^{2,3}$ and a superscan on ${ }^{99 \mathrm{~m}} \mathrm{Tc}-\mathrm{MDP}$ scintigraphy is seen in advanced disease. Superscans have also been described with ${ }^{18}$ F-FDG PET/CT in metastatic cancer. ${ }^{4,5}$ It accumulates in the primary tumor and metastatic soft tissue and skeletal disease. ${ }^{6}$ Normal, unaffected bone demonstrates only mild or absent tracer accumulation. ${ }^{7,899 \mathrm{~m}}$ Tc-MDP whole-body bone scan (B) performed within 24 hours of ${ }^{68} \mathrm{Ga}$-PSMA PET/CT also demonstrated diffusely increased skeletal accumulation, which was less intense than that seen on the PET/CT images. 


\section{References}

1. Kamaleshwaran KK, Mittal BR, Harrisankar CN, et al. Predictive value of serum prostate specific antigen in detecting bone metastasis in prostate cancer patients using bone scintigraphy. Indian J Nucl Med. 2012;27:81-84.

2. Bubendof L, Schopfer A, Wagner $U$, et al. Metastatic patterns of prostate can-cer: an autopsy study of 1,589 patients. Hum Pathol. 2000;31:578-583.

3. Taichman RS, Loberg RD, Mehra R, et al. The evolving biology and treatment of prostate cancer. JClinInvest. 2007;117:2351-2361.

4. Bailly $M$, Besse $H$, Kerdraon R, et al. 18F-FDG PET/CT superscan in prostate cancer. Clin Nucl Med. 2014;39:912-914.

5. Chia-Yang L, Yu-Wen C, Chin-Chuan C, et al. Bone metastasis versus bone marrow metastasis? Integration of diagnosis by $18 \mathrm{~F}$-fluorodeoxyglucose posi-tron emission/computed tomography in advanced malignancy with super bone scan: two case reports and literature review. Kaohsiung J Med Sci. 2013;29: 229-233.

6. Afshar-Oromieh A, Malcher A, Eder M, et al. PET imaging with a [68Ga]galliumlabelled PSMA ligand for the diagnosis of prostate cancer: biodistribution in humans and first evaluation of tumour lesions. Eur J Nucl Med Mol Imaging. 2013;40:797798.

7. Afshar-Oromieh A, Zechmann CM, Eder M, et al. Comparison of PET imag-ing with a 68Ga-labelled PSMA ligand and 18F-choline-based PET/CT for the diagnosis of recurrent prostate cancer. Eur J Nucl Med Mol Imaging. 2014;41:11-20.

8. Mennweiler $S$, Amersdorfer $P$, Trananoski $S$, et al. Heterogeneity of prostate specific membrane antigen (PSMA) expression in prostate carcinoma with distant metastasis. Pathol Oncol Res. 2009;15:167-172. 